\title{
Modelling the dynamic thermal response of turbulent fluid flow through pipelines
}

\author{
Saleh Salavati Meibodi ${ }^{1}$, Simon Rees ${ }^{1}$, Dongmin Yang ${ }^{1}$ \\ ${ }^{1}$ School of Civil Engineering, University of Leeds, Leeds, LS2 9JT, UK
}

\begin{abstract}
The transient behaviour of pipe systems is important in many forms of thermal system such as domestic hot water, building heating, cooling and district thermal networks. In this study, different approaches to modelling the dynamic thermal response of pipelines are investigated through applying three forms of discretized one-dimensional flow and heat transfer model. These were further compared with fully three-dimensional finite volume method (FVM) calculations. Firstly, the models were examined to predict the pipe thermal response considering the thermal capacity and longitudinal dispersion of turbulent fluid flow to step changes in the inlet temperature of a ideally insulated pipe. A model is proposed combining features of plug-flow n-continuously stirred tanks and treatment of the nodes to take into account the effect of thermal capacitance of the pipe wall as well as the convective heat transfer from the pipe outer surface. The results elucidated that the proposed model is not only able to capture the outlet temperature changes due to a step change in the very good agreement against the detailed 3D model but also offers advantages in computational cost compared with the 3D model. The proposed model can be simply implemented in dynamic system simulation tools. The model is to be extended to include dynamic ground heat transfer effects.
\end{abstract}

\section{Introduction}

Understanding and representing the dynamic thermal behaviour of pipes can be considered essential in simulating heating system operation, specifically in situations that short timescale dynamic effects are significant. Often, in the simulation of heating systems, the heat carrier fluid flow through pipes is treated as an ideal fluid with the uniform velocity profile i.e. plug flow. The dynamic transport of heat, as well as the thermal capacity of the fluid, are also often ignored. These assumptions can be deemed correct if the medium and long term simulations are of interest and the pipe lengths are relatively short, but for short timescale simulations and/or where the pipe length is relatively long, cannot be considered accurate.

In a real system, a simple temperature change at the inlet propagated through the pipe is diffused according to the shape of the velocity profile. This physical phenomenon needs to be considered particularly for systems with inlet temperature fluctuations in which case the thermal response at the outlet experiences damping and time lag. This time delay can play a significant role in long pipes with the transit time of higher than a few minutes: possibly more than 30 minutes in district heating systems. These diffusive effects are further compounded when heat transfer occurs in the radial direction, e.g. heat loss from hot water supply pipes or into the ground in district heating.

For dynamic simulations of fluid flow through a pipe, a number of approaches have been proposed (van der Heijde et al., 2017). The propagation of fluid flow through a pipe can be modelled by so-called the node method (Pálsson et al., 1999). In this approach, the heat propagation of the fluid flow is modelled by taking into account only two nodes, the inlet and outlet temperatures, and the time delay due to the transporting the fluid between these two nodes depending on the fluid velocity. Based on the temperature at the inlet node, the pipe wall temperature and mass flow rate, the temperature of the outlet node is calculated and updated from one node to the another by solving energy equation for each node through the pipe. In this method, the heat capacities of fluid and pipe wall are taken into account, and the model can be implemented to deal with dynamic heat losses to surroundings e.g. ground. This model has been implemented to modelling pipelines in district heating systems and shows a good agreement with measurement data (Sartor and Dewalef, 2017). However, since in this model, the fluid flow is assumed as a "plug" flow, the model is not able to capture short timescale fluid dynamic effects.

Another method of modelling the fluid flow through a pipe is to use Finite Element Method (FEM) and Finite Volume Method (FVM) numerical methods. Gabrielaitiene et al. (2008) evaluated the FEM and 
node models for modelling the heat propagation in the district heating pipelines, and compared this method with the node model and the measurement data. They concluded the models have limitations in the prediction of the peak values and temperature response time of the heat wave through the pipe. Dalla Rosa et al. (2013) investigated the transient heat transfer in twin pipes. They proposed a modified node model and validated it against the FVM model and measurement data. It was shown both the detailed FVM model and the proposed model can predict the pipe outlet temperatures when step changes or sinusoidal changes are imposed in the inlet temperature in comparison with the experimental data. Such numerical approaches are very computationally demanding, however.

Another approach to simulate the dynamic responses of pipes is to modelling the dispersion of the fluid flow by applying a one-dimensional advection-dispersion equation, the so-called the Axial Dispersion Plug Flow (ADPF) model. This can be approximated by discretizing the pipe into a series of well-mixed cells with uni-directional flow from the inlet and solving the energy balance for each cell in turn. This approach is implemented to modelling the dynamic thermal response of conduits (Hanby et al., 2002), and borehole heat exchangers (Rees, 2015).

In the present study, a combination of nodal model and a modified approximation of ADPF is proposed for modelling the dynamic thermal responses of long pipes with heat losses to the surroundings. In this method, the fluid flow is represented by the discretized model named the plug flow N-continuously stirred tanks (PFNCST) model. In this model, introduced by Skoglund and Dejmek (2008), the fluid flow is modelled by combining a plug flow model (to represent the time lag accurately) and a series of continuously stirred tanks to represent the axial difusion processes. It is demonstrated that this model is able to accurately predict the dynamic responses to a step change in the fluid flow concentration compared to the exact solution of the ADPF model and is also more computationally efficient compared with other models (Skoglund and Dejmek, 2008). The model presented by Skoglund and Dejmek (2008) was intended to model diffusion of different chemical species in the pipe rather than heat but in this case we model heat transfer to calculate fluid temperatures. The model further allows calculation of radial heat transfer to the environment.

The primary motivation behind this study is to develop a model which is able to accurately predict the dynamic thermal response of pipes with heat losses, and capture short timescale dynamic effects, also efficient in terms of the computational cost and so can be simply implemented in modeling the operation of large and complex thermal systems over annual peri- ods, e.g. district heating systems.

\section{Model Development}

\section{Three-Dimensional Numerical Model}

In this work, a three-dimensional pipe with turbulent fluid flow with conjugate forced convection heat transfer has been modelled using the OpenFOAM library and is intended as a reference model for cases with heat transfer. This model is developed to investigate the thermal dynamic response of a pipe to a step change in the pipe inlet. The flow is assumed incompressible, Newtonian, three-dimensional and (low-Reynolds Number) turbulent. The fluid is deemed to have a uniform inlet velocity profile and uniform inlet temperate.

This numerical model uses the finite volume method to numerically solve the governing Navier-Stokes and energy equations. In order to solve the equations in non-compressible form, the PIMPLE algorithm which is a combination of PISO (Pressure Implicit with Splitting of Operator) and SIMPLE (SemiImplicit Method for Pressure-Linked Equations) is implemented. The first-order upwind scheme is exploited to discretize the temporal term, and a secondorder scheme is employed to discretize the convection and diffusion terms of the governing equations. To model the turbulent flow in the pipe, the well-known $k-\omega$ Shear Stress Transport- SST model is applied in this work. The energy equation is solved simultaneously in both solid and fluid domains to examine the combination of convection and conduction effects.

The pipe geometry is discretised using a threedimensional structured mesh. Since near the pipe wall the temperature and velocity gradients are high, the dimension of cells has been reduced from a pipe centre to the pipe wall to capture the gradients near the pipe wall. The multi-block structured mesh for the pipe is shown in Fig. 1. The independence of results to the mesh sizes is investigated in the following section.

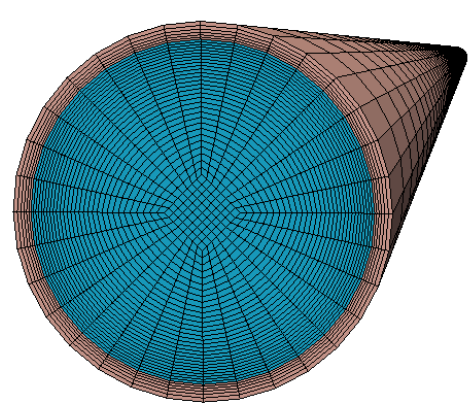

Figure 1: A Multi-block structured Mesh representing a pipe.

\section{Residence Time Distribution}

The Residence Time Distribution (RTD) is defined as the distribution with time of transported scalar vari- 
ables (chemical species or heat) as they pass through a particular point (e.g. outlet) of a continuous flow system. RTDs are widely used to analyse chemical engineering problems and are approximated using a form dispersion model or tanks in series models (Ham and Platzer, 2004). The RTD of a system is commonly expressed as a function $F(t)$ representing the fraction of the fluid scalar state variable (chemical species or heat) at the pipe outlet for fluid flow at a given time: often plotted as a so called F-diagram.

It has been demonstrated that the shape of F-diagram depends on Reynolds number and the ratio of length to a diameter only affects the eddy diffusivity. Hanby et al. (2002) implemented the analytical solution to finding the optimum number of tanks in series to modelling dynamic thermal response of conduits. The same approach has been used by He (2012) for simulation of dynamic thermal response in a twodimensional borehole heat exchanger model. Ham and Platzer (2004) proposed a semi-empirical formulation for calculating residence time distribution based on characteristic parameters that are determined from experimental results and this has been used as a reference in other work (Adeosun and Lawal, 2010). In this model, the F-diagram can be calculated according to,

$$
F(\tau)=\left[1-\frac{\tau_{k}^{N}}{\tau^{N}}\left(1-\frac{\tau}{\tau_{\max }}\right)^{N}\right]^{8}
$$

where,

$$
\tau_{k}=\frac{\tau_{\min } \tau_{\max }}{\tau_{\max }-\tau_{\min }}
$$

The $\tau_{\min }$ and $\tau_{\max }$ parameters are the experimental minimum and the maximum dimensionless residence time of the tracer and $\tau$ is dimensionless residence time. $\mathrm{N}$ is also a model parameter that is obtainable according to the experimental minimum and maximum dimensionless residence times. We have used this model as a reference in validating models with perfectly insulated pipes below.

\section{Modelling dynamic responses of perfectly in- sulated pipes}

In this study, the approach to modelling dynamic response of heat transfer fluid in pipelines is to utilize a discretized model of the fluid flow through the pipe which takes into account the thermal capacity and longitudinal dispersion. It is demonstrated that the dispersion of turbulent fluid flow in pipes for chemical concentration species as well as heat can be successfully modelled by employing axial dispersion plug flow (ADPF) model. With no source term and no chemical reaction, the one-dimensional of the ADPF model can be written as below (Skoglund and Dejmek, 2008),

$$
\frac{\partial C(x, t)}{\partial t}+v \frac{\partial C(x, t)}{\partial x}-D \frac{\partial^{2} C(x, t)}{\partial x^{2}}=0
$$

where $C(x, t)$ is volume chemical concentration and $D$ is the diffusion coefficient which depends on velocity profile and Reynolds number. For the calculation of diffusion coefficient an empirical relation (Wen and Fan, 1975) in terms of Peclet Number Pe (the ratio of advective transport rate to the diffusive transport rate) can be applied as follows,

$$
\frac{1}{P e}=\frac{D}{L v}=\frac{2 r_{p}}{L}\left(3 \times 10^{7} R e^{-2.1}+1.35 R e^{-0.125}\right)
$$

Where $L$ is the length of the pipe and $r_{p}$ is the inner radius of the pipe. Eq.3 can be converted to a onedimentioanl thermal convection-diffuson, if $C(x, t)$ is replaced with $T(x, t)$. Applying the laplace trasform to Eq.3, for the exit concentration at the $x=L$, the transfer function can be given by (Skoglund and Dejmek, 2008),

$$
G_{A D P F}(s)=e^{P e / 2} e^{-(P e / 2) \sqrt{1+(4 / P e) \tau s)}}
$$

Due to mathematical difficulties of dealing with Eq.5, a number of discrete approximations have been proposed in the literature. The well-known approximation to the ADPF model is referred to as $\mathrm{N}$ continuously stirred tank (N-CST) model. In this model, the pipe is represented by series of well-stirred tanks and is effectively a one-dimensional finite volume or finite difference representation. This model has been utilized for modelling dynamic thermal response of conduits (Hanby et al., 2002) and a borehole heat exchanger (He, 2012). However, it is shown that this model is sensitive to the number of tanks, and also tends to over predict the diffusivity of the dynamic responses. To deal with this issue Wen and Fan (1975) derived an expression for the optimal number of tanks for the best approximation to the true ADPF model behaviour.

$$
N_{C S T}=\frac{v L}{2 D}=\frac{P e}{2}
$$

Skoglund and Dejmek (2008) proposed an improved model that is a combination of a plug flow representation in series with an N-continuously stirred tanks model (denoted PFNCST). In this model, a simple time delay is introduced as a plug flow at the inlet before the fluid enters the series of continuously stirred tanks. This approach shows better accuracy compared to the analytical solution of the ADPF equations compared to the N-CST model. The results are less sensitive to the choice of the number of tanks. Moreover, the number of tanks required in PFNCST model is considerably less than the former model and so is computationally advantageous.

In this model, the transit of fluid flow through a pipe is divided between two types of elements. The first plug flow element has an associated transport time delay $\left(\tau_{0}\right)$ and the remaining transit time is associated with $N$ ideal tanks elements $\left.\left(N \tau_{N}=\tau-\tau_{0}\right)\right)$, as shown in fig. 2. The heat balance equation on these 


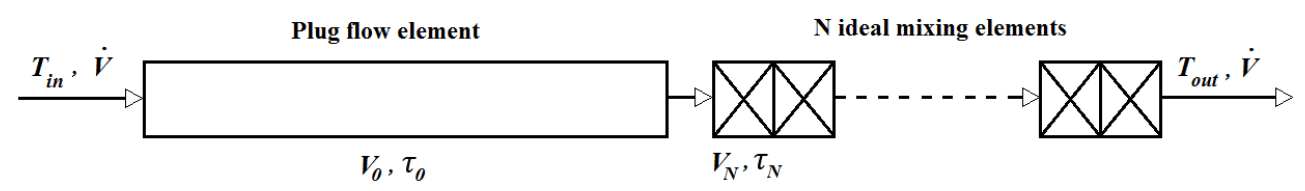

Figure 2: A representation of fluid flow through a pipe using the PFNCST approach.

two elements can be written as,

$$
\rho C_{p} V_{N} \frac{\partial T_{i}}{\partial t}+\rho C_{p} \dot{V}\left(T_{i}-T_{i-1}\right)=0
$$

Where $V$ and $\dot{V}$ are the volume of each tank and volume flow rate of the fluid, respectively. Considering the time delay in plug flow element, the inlet temperature for the $\mathrm{N}$ ideal tanks part is $\left(T_{i=0}(t)=\right.$ $\left.T_{i n}\left(t-\tau_{0}\right)\right)$. Skoglund and Dejmek (2008) demonestrated that $\tau_{N}$ can be calculated by Eq. 8 with very good agreement with ADPF model.

$$
\tau_{N}=\tau \sqrt{\frac{2}{N P e}}
$$

Accordingly, the volume of each tank and plug flow element as well as $\tau_{0}$ can be determined for a given number of tanks. This model has been used by Rees (2015) for simulation of the thermal responses of borehole heat exchangers. It was demonstrated that this model was able to accurately capture some of the short timescale features of experimental fluid temperature data. However, the heat losses from pipe surfaces to surroundings have not been considered.

\section{Modelling dynamic responses of uninsulated pipes}

In the current work we have sought to apply and extend the PFNCST model for modelling dynamic thermal responses of pipe where there is heat exchange with the surroundings. To this end, the finite difference method is used for the calculation of the fluid temperatures and pipe wall temperatures by addition of a further node to take into account the thermal capacity pipe wall and to represent the radial temperature gradients as illustrated in Fig.3. This seems a reasonable approximation for relatively thin walled pipes. The model can be described by two heat balance differential equations for the fluid and wall nodes as follows,

$$
\begin{aligned}
\rho_{f} C_{p, f} V_{N} \frac{\partial T_{f, i}}{\partial t}= & \rho_{f} C_{p, f} \dot{V}\left(T_{f, i-1}-T_{f, i}\right)- \\
& h_{f} A_{i}\left(T_{f, i}-T_{w, i}\right) \\
\rho_{w} C_{p, w} V_{N} \frac{\partial T_{w, i}}{\partial t}= & h_{f} A_{i}\left(T_{f, i}-T_{w, i}\right)- \\
& h_{a} A_{i}\left(T_{w, i}-T_{a}\right)
\end{aligned}
$$

At each node, the fluid temperature obtained from the PFNCST model is updated in each time step by calculation of heat losses according to the fluid and

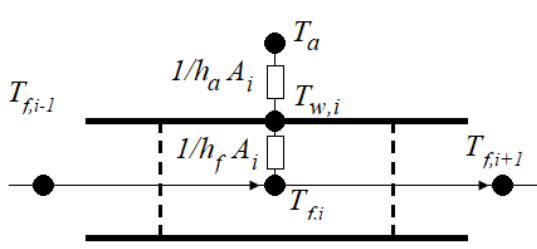

Figure 3: The representation of heat transfer from each well-mixed fluid element to the surroundings.

pipe wall thermal capacities, the heat transfer coefficient between fluid and the inner layer of the pipe wall, and the heat transfer coefficient from the outer layer of the pipe wall to the surroundings.

In this model, it is necessary to define the proper pipe inner surface boundary condition and a relationship between the wall temperature and the fluid temperature at the inlet and outlet of each tank element. A convenient way to formulate the relationship between these temperatures would be to use the arithmetic mean of the inlet and outlet temperatures and apply it in a convective boundary condition. However, due to the long 'virtual' length of the Plug flow element in the model at typical flow rates, we found this approach performed poorly. Another approach is to make an analogy with an evaporating-condensing heat exchanger. In this approach, a pipe element is assumed to have the same wall temperature along its length and considered as a heat exchanger that is characterized by an effectiveness parameter $\epsilon$, and the Number of Transfer Units NTU. The relationship between effectiveness parameter which is the ratio of the actual fluid heat transferred to the maximum possible heat transfer is,

$$
\epsilon=\frac{\dot{m} C_{p}\left(T_{\text {in }}-T_{\text {out }}\right)}{\dot{m} C_{p}\left(T_{\text {in }}-T_{p}\right)}
$$

and using the evaporating-condensing isothermal wall assumption, the relationship between effectiveness and NTU can be expressed as,

$$
\epsilon=1-e^{-N T U}
$$

where,

$$
N T U=\frac{\pi D L h_{p}}{\dot{m} C_{p}}
$$

and using well-known Dittus-Boelter equation, the pipe convection coefficient, $h_{f}$ can be calculated as,

$$
h_{f}=\frac{0.023 \operatorname{Re}^{0.8} \operatorname{Pr}^{0.4} \lambda_{f}}{D}
$$




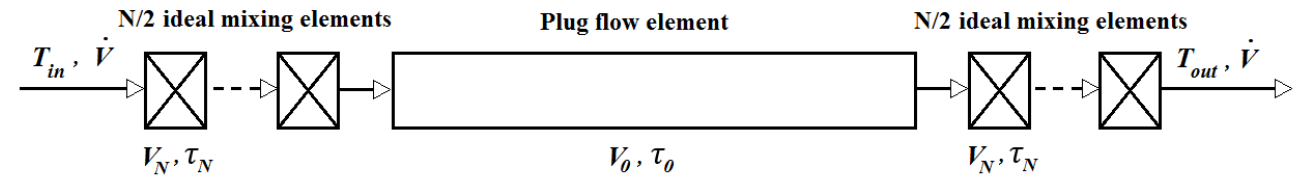

Figure 4: A diagram of the proposed modelling fluid flow through a pipe.

Where $\lambda_{f}$ and $\operatorname{Pr}$ are the thermal conductivity and Prandtl number of the fluid, respectively. Considering Eq.11, the heat balance at the pipe boundary can be written:

$$
\epsilon \dot{m} C_{p}\left(T_{i n}-T_{p}\right)=h_{a} A\left(T_{p}-T_{a}\right)
$$

Using Eq.11 and 15, the pipe wall and outlet fluid temperatures can be calculated explicitly for a given inlet temperature at each time step. We found that simply applying this approach to the PFNCST model proposed by Skoglund and Dejmek (2008) did not give realistic temperature responses where there was heat transfer to the environment. As the initial (and often longest) element in the pipe represents plug flow but not heat transfer, the effects of heat transfer are also delayed. With a step change in inlet temperature this is particularly unrealistic.

In this study, the a arrangement of plug flow element and well-stirred elements in the PFNCST model has been proposed to properly calculate the dynamic heat losses along the pipe. To this end, the number of ideal mixing elements was divided into two sections placed at the inlet and outlet of the pipe with the plug flow element between as shown in Fig.4. The volume of each tank element and the time required for passing the fluid flow through each tank are obtained in the same way as the PFNCST model. This arrangement modifies the inlet temperature experienced by the plug flow element to reflect some of the dynamic heat losses occurring along the pipe. The ability of the proposed PFNCST model in the prediction of the outlet temperature of a pipe with heat losses is presented and discussed in the following section.

\section{Results and discussions}

\section{Turbulent velocity profile}

To validate the $3 \mathrm{D}$ model developed in this work and that we later use as a reference model, experimental data from the literature have been used. Due to the importance of velocity profile in the turbulent pipe fluid flow i.e. Reynolds number is higher than 4000 , on the RTD and dynamic response of pipes we have firstly sought to verify the models predictions of pipe velocity profile. Many researchers have conducted different types of experiments to study fully developed turbulent pipe flow. In the current work, experimental data from particle image velocimetry (PIV) experiments (Eggels et al., 2006; Peng et al., 2018) have been chosen as these are thought to represent the lowest levels of uncertainty: reported to

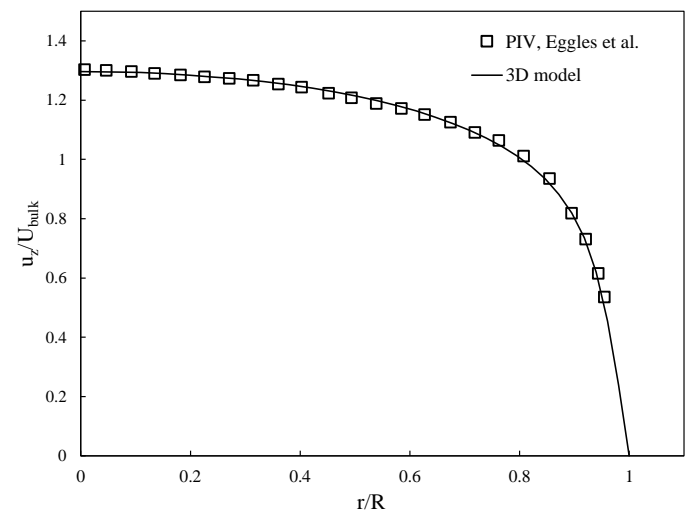

Figure 5: Axial mean velocity profile normalized by the bulk flow velocity.

be less than 2 percent. Fig.5 shows a comparison of predicted and measured mean velocity profile normalized by the bulk flow velocity with respect to the pipe radius for a $10 \mathrm{~m}$ straight pipe at a Reynolds number of 5300. The numerical simulation results show very good agreement with the experimental data over most of the radius. However, near the pipe wall, the simulation shows larger deviations (still less than 1.5 percent RMSE). Eggels et al. (2006) suggested that deviations could be larger in this region of lower velocity due to the fixed sampling intervals in the PIV measurements.

In order to check the mesh size dependency, five mesh sizes were generated to compare velocity profiles. It was observed by increasing cell number from 47,000 to 208,000 , the RMSE decreased from nearly 4.3 to 1.35 percent. Considering computational cost, a mesh with intermediate density $(159,000$ cells $)$ was chosen to model the pipe flow and heat transfer in later calculations.

\section{Residence time distributions}

To validate the $3 \mathrm{D}$ model in terms of predicting the RTD, the semi-empirical model proposed by Ham and Platzer (2004) for a straight pipe has been used as a reference. Based on the experimental conditions in their work, a $9.6 \mathrm{~m}$ straight pipe with $15 \mathrm{~mm}$ diameter was modelled where the mean velocity, Reynolds Number and kinematic viscosity were $0.21 \mathrm{~m} / \mathrm{s}, 4500$ and $7 \times 10^{-7} \mathrm{~m}^{2} / \mathrm{s}$, respectively. The measured mean residence time is reported as $45.7 \mathrm{~s}$, and the minimum and maximum residence times were measured as $38.89 \mathrm{~s}$ and $77.01 \mathrm{~s}$, respectively. Applying these 
experimental conditions and using the semi-empirical model, the F-diagram can be plotted based on Eq. 1. Fig. 6 presents this F-diagram generated using the $3 \mathrm{D}$ model and the semi-empirical model according to the experimental data. A very good level of agreement between the semi-emperical model and the 3D model developed in this work has been demonstrated. The minor deviations may be due to the uncertainty in minimum and maximum residence times as these are important values in calculation of the RTD.

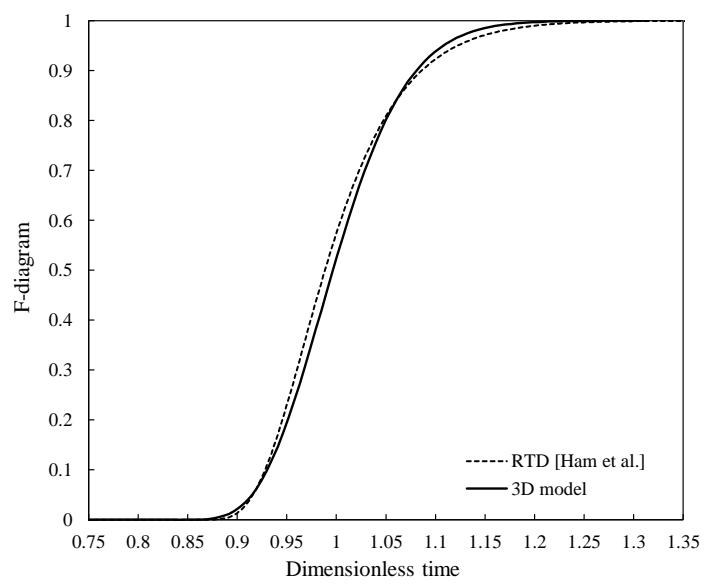

Figure 6: Comparison of F-diagram calculated by using the $3 D$ model and the semi-emperical model.

\section{Dynamic response of the ideally insulated pipe to a step change}

To investigate the proposed models in the simulation of the dynamic responses of the ideally insulated pipe, the results of the two discretized models, the NCST and PFNCST, have been compared to the exact solution of the ADPF model. To this end, responses in a $10 \mathrm{~m}$ straight pipe of $15 \mathrm{~mm}$ diameter have been studied. The water flow velocity and Reynolds number were $0.5 \mathrm{~m} / \mathrm{s}, 9700$ respectively. In this case, the inlet temperature of the pipe is imposed to a step change of $60{ }^{\circ} \mathrm{C}$, from $20^{\circ} \mathrm{C}$ to $80^{\circ} \mathrm{C}$, while the initial temperatures of the water and pipe wall are $20^{\circ} \mathrm{C}$. It is considered that heat transfer at the pipe wall is zero i.e. the R-value is infinity, so an adiabatic boundary condition is applied for the inner surface of the pipe. Fig. 7 displays the variations of the outlet temperatures responses to a step change calculated by three models including the NCST, PFNCST and exact solution of the ADPF models to a step change. Based on Eq. 6, the optimal number of tanks in the NCST model is calculated 661 , and two tank numbers, i.e. 16 and 34, are selected for the PFNCST model. The one-dimensional of the advection-dispersion equation (ADPF) is numerically solved for this case by applying the Eq.5.

It is seen the PFNCST model with considerably fewer number of tanks shows better agreements with the exact solution of ADPF in the prediction of the out- let temperature and the NCST model tends to overpredict the diffusion in responses to a step changes; as reported by Rees (2015) and Hanby et al. (2002). Moreover, it is found the variation of the amount of tanks in the PFNCST model does not have noticeable effects on the accuracy of the prediction of dynamic responses i.e. the model is robust in this respect.

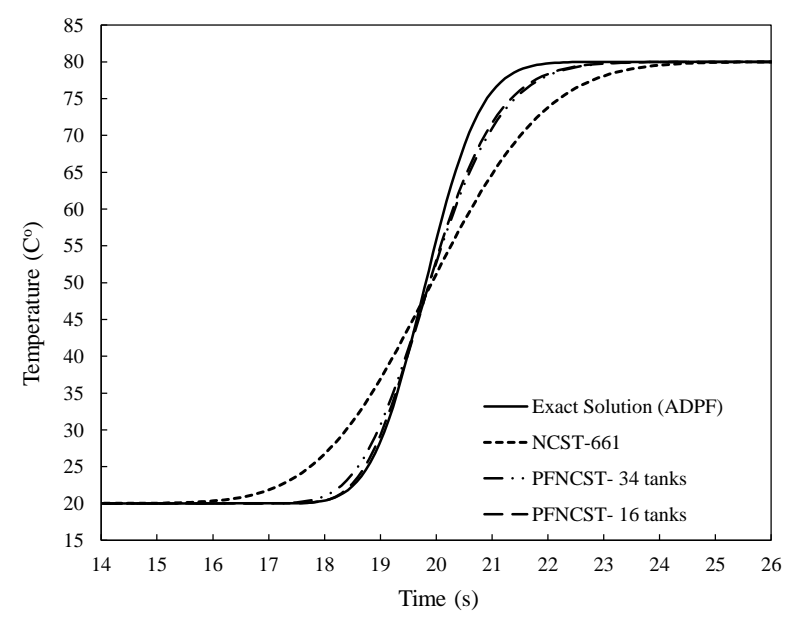

Figure 7: Comparison between the outlet temperature responses to a step change calculated by the NCST, PFNCST models and the exact solution of the ADPF model for an insulated pipe.

\section{Dynamic response of the uninsulated pipe to a step change}

To evaluate the ability of the different approaches to modelling the dynamic thermal response of uninsulated pipe to a step change, again a $10 \mathrm{~m}$ straight pipe of $15 \mathrm{~mm}$ diameter has been modelled with the same velocity and Reynolds number. In this case the heat losses along the pipeline due to temperature differences between the pipe wall, the surroundings and fluid flow are taken into account. Note that the inner pipe surface heat transfer coefficient is calculated based on Eq. 14 and the outer pipe surface heat transfer coefficient is considered constant and equal to $10 \mathrm{~W} / \mathrm{m}^{2}$.K.

Fig. 8 displays comparisons between the three models of interest - the NCST, modified PFNCST and the 3D numerical model - in the prediction of outlet temperature response to a step change in the presence of radial heat losses. The data in Fig. 8 show the modified PFNCST model is in good agreement with the detailed 3D model results and slightly better than is the NCST model with considerably lower tanks. The modified PFNCST model can anticipate the time of the initial increase of the outlet temperature and can also predict the rising trend in temperature at the pipe outlet, compared with the detailed 3D model. There may be some minor differences between the models as the outlet temperature approaches the steady condition. However, we suggest this is not usu- 
ally the aspect of the response that is of most interest or significance.

To assess the validity of the modified PFNCST model further, the models have been compared with higher and lower fluid velocities $(0.7 \mathrm{~m} / \mathrm{s}$ and $0.3 \mathrm{~m} / \mathrm{s})$ as shown in Fig. 9 and 10. These flow velocities correspond to Reynolds Numbers of 13600 and 5800 . Similar levels of agreement in terms of predicted response are shown as in Fig. 8. However, slightly more sensitivity to the choice of number of tanks in the PFNCST model than cases without heat loss was demonstrated.

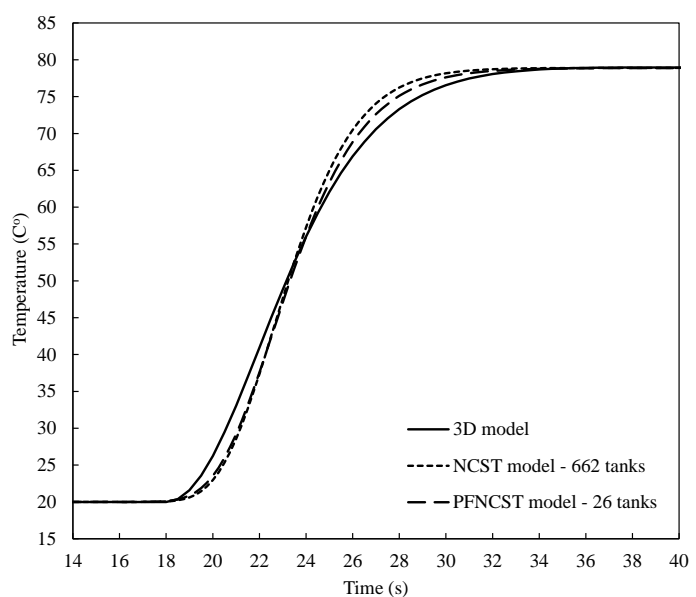

Figure 8: Outlet temperature response calculated by the NCST, PFNCST and 3D model for the uninsulated pipe at $R e=9700$.

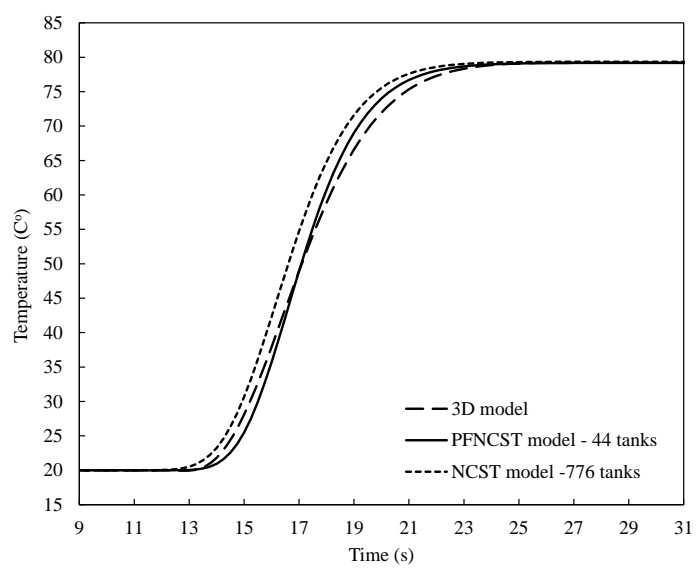

Figure 9: Outlet temperature response calculated by the NCST, PFNCST and 3D model for the uninsulated pipe at $R e=13600$.

By comparing the differences in predicted temperatures with different numbers of tanks using the RMSE, optimal numbers of tanks were obtained for each Reynolds number. It was found that a lower number of tanks causes a sharp thermal response to a step change (less diffusive) and vice versa. This is a result of the diffusion processes being more complex

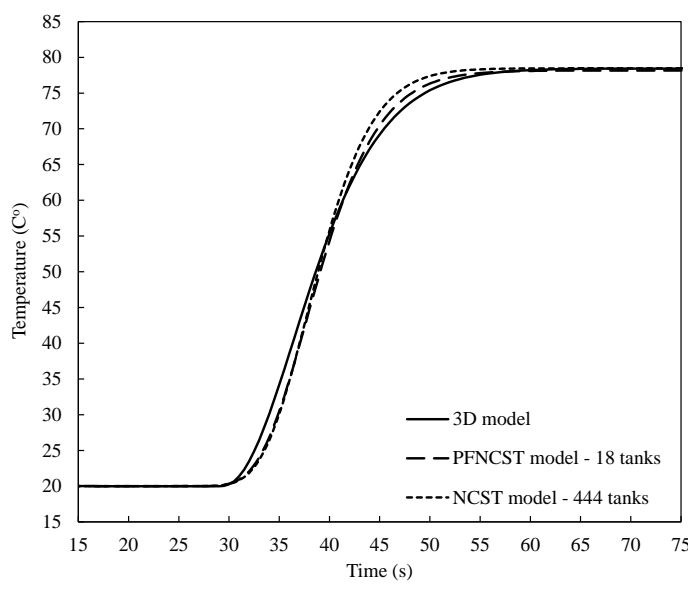

Figure 10: Outlet temperature response calculated by the NCST, PFNCST and 3D model for the uninsulated pipe at $R e=5800$.

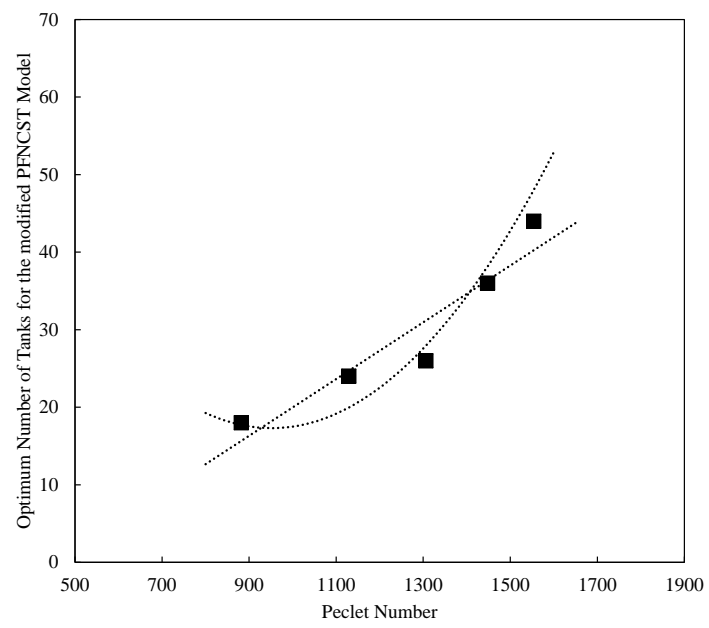

Figure 11: Variation of the optimal number of tanks for the PFNCST and Pe number.

in cases with heat transfer through the pipe wall: heat is diffused both axially and radially. In these calculations we are also seeking to include the thermal mass of the pipe material. Consequently we have investigated whether a rule or correlation can be identified to guide the appropriate choice of the number of tank elements in cases with pipe wall heat transfer.

In a similar way to the NCST model, we have found that the optimal number of tanks is well correlated with Peclet Number. We have arrived at the preferred values of number of tanks for a range of flow conditions by carrying out a parameter estimation based on the F-diagram and corresponding RMSE measure. This has resulted in the data shown in Fig. 11 where $\mathrm{Pe}$ is calculated based on Eq. 4. A well defined trend is evident. This might be approximated by a linear trend or possibly a second order polynomial. Although the model is not strongly sensitive to this number, using the optimal number of tanks could be important in the cases such as where there are high 
frequency dynamics and important control system interactions.

It is worth noting that the calculation time for simulating of fluid flow in the pipe in the 3D models (with $20 \mathrm{CPU}$ cores) is more than twenty times that of the proposed model (serial). It is also notable that the number of tanks in the modified PFNCST is considerably lower than NCST model and so less computationally demanding. This feature could be significant in modelling a very long pipe, such as pipe networks in district heating systems.

\section{Conclusions and future work}

A three-dimensional model has been developed using the Finite Volume Method to modelling the dynamic thermal responses of turbulent fluid flow through pipes. This model has been shown to be in a good agreement with experimental data in terms of the prediction of velocity profiles and residence time distribution (RTD) and has been used as a reference model in parametric studies and development of reduced order models. Moreover, a new numerical model combining nodal and the PFNCST approaches has been proposed for modelling the dynamic thermal response of pipe systems considering heat transfer to the environment. This model is able to simulate the heat propagation through the pipe due to a step change at the inlet of the pipe, and capture short-time dynamic effects with same level of accuracy of the detailed three-dimensional model as well as the effect of the pipe material and external heat exchange.

A heat exchanger analogy is used to define the relationship between inlet, outlet and the pipe wall temperatures in each element. The outlet temperature of the plug flow and continuously stirred tank elements can be calculated explicitly at each time step resulting in a computationally efficient model. The model has a modest level of sensitivity to the number of tanks used at different Reynolds Numbers and we have proposed that a simple correlation with Peclet Number can be applied to allow this to be determined. It is proposed in future work, the model to be validated with experimental data over a wider range of experimental conditions, i.e. different Reynolds numbers and pipe geometries. Our primary interest is in district heating network simulation and we proposed to extend the representation of heat transfer to include transient heat transfer through the ground and also between adjacent flow and return pipes.

\section{References}

Adeosun, J. T. and A. Lawal (2010). Residence-time distribution as a measure of mixing in T-junction and multilaminated/elongational flow micromixers. Chemical Engineering Science 65(5), 1865-1874.

Dalla Rosa, A., H. Li, and S. Svendsen (2013). Modeling transient heat transfer in small-size twin pipes for end-user connections to low-energy district heating networks. Heat Transfer Engineering 34(4), 372-384.

Eggels, J. G. M., F. Unger, M. H. Weiss, J. Westerweel, R. J. Adrian, R. Friedrich, and F. T. M. Nieuwstadt (2006). Fully developed turbulent pipe flow: a comparison between direct numerical simulation and experiment. Journal of Fluid Mechanics 268(-1), 175.

Gabrielaitiene, I., B. Bøhm, and B. Sunden (2008). Evaluation of approaches for modeling temperature wave propagation in district heating pipelines. Heat Transfer Engineering 29(1), 45-56.

Ham, J.-H. and B. Platzer (2004). Semi-Empirical Equations for the Residence Time Distributions in Disperse Systems - Part 1: Continuous Phase. Chemical Engineering \& Technology 27(11), 11721178.

Hanby, V., J. Wright, D. Fletcher, and D. Jones (2002). Modeling the dynamic response of conduits. HVAC \& R Research 8(1), 1-12.

He, M. (2012). Numerical modelling of geothermal borehole heat exchanger systems. Ph. D. thesis.

Pálsson, H., H. V. Larsen, B. Bøhm, and H. F. Ravn (1999). Equivalent Models for District Heating Systems. Number 1323.

Peng, C., N. Geneva, Z. Guo, and L. P. Wang (2018). Direct numerical simulation of turbulent pipe flow using the lattice Boltzmann method. Journal of Computational Physics 357, 16-42.

Rees, S. J. (2015). An extended two-dimensional borehole heat exchanger model for simulation of short and medium timescale thermal response. Renewable Energy 83, 518-526.

Sartor, K. and P. Dewalef (2017). Experimental validation of heat transport modelling in district heating networks. Energy 137, 961-968.

Skoglund, T. and P. Dejmek (2008). A dynamic object-oriented model for efficient simulation of microbial reduction in dispersed turbulent flow. Journal of Food Engineering 86(3), 358-369.

van der Heijde, B., M. Fuchs, C. Ribas Tugores, G. Schweiger, K. Sartor, D. Basciotti, D. Müller, C. Nytsch-Geusen, M. Wetter, and L. Helsen (2017). Dynamic equation-based thermo-hydraulic pipe model for district heating and cooling systems. Energy Conversion and Management 151 (August), 158-169.

Wen, C. and L. Fan (1975). Models for flow systems and chemical reactors. Chemical processing and engineering. Dekker. 